\title{
Polymorphism in Apolipoprotein C3 (APOC3) and Fatty Acid-Binding Proteins (FABP2) Genes in Nondiabetic Dyslipidemic Patients: A Tertiary Care Hospital-Based Pilot Study
}

\author{
Rashmi Chowdhary $^{1}$ Neha Masarkar ${ }^{1}$ Sagar Khadanga ${ }^{2}$ \\ ${ }^{1}$ Department of Biochemistry, All India Institute of Medical Sciences, \\ Bhopal, Madhya Pradesh, India \\ 2 Department of Medicine, All India Institute of Medical Sciences, \\ Bhopal, Madhya Pradesh, India \\ Address for correspondence Rashmi Chowdhary, MSc, MPhil, PhD, \\ Department of Biochemistry, All India Institute of Medical Sciences, \\ Bhopal, Madhya Pradesh - 462020, India \\ (e-mail: rashmi.biochemistry@aiimsbhopal.edu.in).
}

J Lab Physicians 2022;14:119-124.

\begin{abstract}
Keywords

- APOC3

- dyslipidemia

- FABP2

- nondiabetic

- polymorphism

Context Dyslipidemia is a multifactorial disease in which lipoproteins play an important role as one of the early markers for coronary heart disease (CHD). Mixed dyslipidemia is common in people with diabetes mellitus, but nondiabetic dyslipidemics (NDD) remain unidentified for the risk of developing dyslipidemia and eventually CHD.

Objectives This pilot study attempts to analyze the genetic basis of lipid metabolism alterations, emphasizing the association between fatty acid-binding protein-2 (FABP2Ala54Thr) and apolipoprotein-C3 (APOC3-rs5128) genetic polymorphism, as a risk for developing dyslipidemia and CHD in NDD.

Methods and Design Total 90 subjects-30 DD, 30 NDD, and 30 apparently healthy subjects representing Central India-were included. Biochemical analysis and DNA genotyping were done by polymerase chain reaction restriction fragment length polymorphism.

Statistical Analysis The biochemical parameters were reported as means \pm standard deviation. One-way analysis of variance test was used to compare biochemical parameters of three groups. Chi-squared test was done to compare genotype distributions. The strength of association was assessed by odds ratios (ORs) with 95\% confidence intervals (Cls). All statistical analysis was done using SPSS-PC software and Graph Pad.

Results In NDD, maximum polymorphism was observed followed by DD and least polymorphism was observed in controls. There was a significant association of $A P O C 3 \mathrm{G}$ allele with occurrence of hypertriglyceridemia $(p<0.05)$; however, no such association was found for FABP2 A allele $(p>0.05)$. Logistic regression analysis revealed APOC3 polymorphism to be significantly associated with dyslipidemia $(\mathrm{OR}=2.6667,95 \% \mathrm{Cl}=$ $1.0510-6.7663, p=0.0341$ ); no such association was found for FABP2 polymorphism
\end{abstract}

published online July 12,2021
DOI https://doi.org/

10.1055/s-0041-1731949. ISSN 0974-2727.
(C) 2021. The Indian Association of Laboratory Physicians. All rights reserved.

This is an open access article published by Thieme under the terms of the Creative Commons Attribution-NonDerivative-NonCommercial-License, permitting copying and reproduction so long as the original work is given appropriate credit. Contents may not be used for commercial purposes, or adapted, remixed, transformed or built upon. (https://creativecommons.org/ licenses/by-nc-nd/4.0/)

Thieme Medical and Scientific Publishers Pvt. Ltd., A-12, 2nd Floor, Sector 2, Noida-201301 UP, India 
$(\mathrm{OR}=0.4643,95 \% \mathrm{Cl}=0.1641-1.3136, p=0.1347)$. The triglyceride and cholesterol values in individuals with homozygous genotype indicate that genetic study is comparable to the biochemical findings in carriers of polymorphic allele than noncarriers, especially in NDD patients.

Conclusions Pilot study indicates that the presence of $A P O C 3$ gene polymorphism is associated with pro-atherogenic dyslipidemia in nondiabetic patients and may raise risk of CHD. This information could be used for preventive strategies in NDD group that may otherwise go unnoticed.

\section{Introduction}

Dyslipidemia is a key health problem and concern for the clinicians' worldwide. ${ }^{1}$ It is characterized by any or a combination of the following: elevated low-density lipoprotein (LDL-C; > 130md/dL), elevated total cholesterol (CHOL; $>200 \mathrm{mg} / \mathrm{dL}$ ), elevated triglyceride (TG; > 150mg/dL), or low high-density lipoprotein (HDL-c; $<40 \mathrm{mg} / \mathrm{dL}$ in men and $<50 \mathrm{mg} / \mathrm{dL}$ in women $)^{2}$ These conditions play a major role in the progression of coronary heart diseases (CHD). Hypercholesterolemia is a key independent modifiable risk factor for CHD and leading cause of death worldwide, India alone contributing to almost one-fifth (18.6\%) of the global CHD burden. ${ }^{3}$ It accounts for $24 \%$ of all deaths among adults aged between 25 and 69 years. ${ }^{4}$ Although plasma lipid concentrations are strongly influenced by nutritional and lifestyle choices, 50 to $80 \%$ of the variation is influenced genetically. ${ }^{5}$

Single nucleotide polymorphism in a few genes is associated with high triglyceride (TG) and CHOL levels significantly contributing to dyslipidemia. ${ }^{6} \mathrm{~A}$ gene associated with $\mathrm{TG}$ and CHOL level is the gene encoding for fatty acid-binding proteins (FABP2) located at the chromosome 4q2-q31, another gene apolipoprotein $\mathrm{C} 3$ gene $(A P O C 3)$ located on the chromosome $11 \mathrm{q} 23$, allele ${ }^{*} 3238 \mathrm{C}$ is also involved in the regulation of plasma TG levels. ${ }^{7,8}$

The circulating lipid biomarkers, mainly polymorphism APOC3 (rs5128) and FABP2 (rs1799883), had been independently studied in diabetic patients, but the associations between these biomarkers and occurrence of dyslipidemia in nondiabetic patients had rarely been studied. There is no information regarding the genetic influence of dyslipidemia and their relations with biochemical estimations of circulating lipoproteins in the population representing Bhopal city (Central India). The present study explored the correlations of serum lipid levels and variations in the genes governing lipid metabolism very critically to find out their role with respect to increased serum lipid levels in the nondiabetic dyslipidemics (NDD) and how different they will be in comparison to the diabetic patients.

\section{Subjects and Methods}

\section{Ethics Statement}

Ethics statement approval of the Institutional Ethics Committee was obtained before study commencement (IHEC approval number - LOP/2017/IM0147).

\section{Study Population}

The study was conducted on unselected population (in terms of their gender, occupation, ethnicity, socioeconomic status) coming to Dyslipidemia Clinic of Department of General Medicine. Clinical history was identified by physician (January 2017 to September 2018). To identify the study population, risk assessment was done based on the following inclusion criteria: advancing age (35-70 years), diabetes mellitus, hypertension, family history of dyslipidemia; exclusion criteria: polycystic ovarian syndrome, hypothyroidism, cerebral trauma, autoimmune diseases, tumors, and infectious diseases.

Written informed consent was obtained from all study subjects in English/Hindi language. All participants were adult between the age 35 to 70 years under the inclusion criteria and few were on statins (TG-lowering medication).

\section{Groups}

Based on the inclusion and exclusion criteria, 90 subjects were selected as study population and divided into three groups of 30 each (following a flat rule of thumb for pilot studies): Group 1: men and women aged between 35 and 70 years who are DD; Group 2: men and women aged between 35 and 70 years who are NDD; Group 3: men and women aged between 35 and 70 years who do not have diabetes and dyslipidemia ( $\mathrm{C}$ or control group).

\section{Biochemical Analysis and Genotyping}

For lipid profile tests, $5 \mathrm{~mL}$ of fasting venous blood sample was collected in serum vial (yellow vial), and for fasting blood sugar (FBS) tests, $1 \mathrm{~mL}$ of blood was collected in fluoride vial (gray vial). For DNA analysis, $1 \mathrm{~mL}$ of blood was extracted in ethylenediaminetetraacetic acid vial (purple vial). Lipid profile tests, plasma glucose (FBS), and glycosylated hemoglobin (data not shown) were determined to confirm and classify the study population into DD, NDD, and C (healthy controls). All the biochemical analysis was done in automated high throughput analyzer Beckman Coulter AU680 (Auto Analyzer). Before starting any estimation, the machine was first standardized, and quality control was run.

Genomic DNA was isolated from peripheral blood samples using organic or phenol-chloroform method. Extracted DNA was run in $1 \%$ agarose gel, in $1 \mathrm{X}$ TAE buffer, for 25 to 30 minutes and visualized on Gel Doc system. Extracted DNA was subjected to polymerase chain reaction (PCR), artificial restriction site was introduced in the DNA. For 
FABP2, primers used were F:5'-TACCGAGTTTTCTTCCCACCC-3', R:5'-TTAAATATCCTGCCAATTTGTGC-3' product size $456 \mathrm{bp}$, the denaturation temperature was $94^{\circ} \mathrm{C}$, the annealing temperature was $59^{\circ} \mathrm{C}$, and extension temperature was $72^{\circ} \mathrm{C}$. For APOC3, primers used were F: $5^{\prime}$ - CCAGTGAAGTTGAGAGGGTG3', R:5'-ACCCACAGAACAGCCTCG-3' product size 501bp, denaturation temperature was $94^{\circ} \mathrm{C}$, the annealing temperature was $62^{\circ} \mathrm{C}$, and extension temperature was $72^{\circ} \mathrm{C}$. The products thus obtained were resolved using $2.5 \%$ agarose gel and then visualized in the Gel-Doc system.

The PCR products were digested with their respective restriction enzymes. For $F A B P 2$, enzyme Hhal was used, the size of the digested fragments $197 / 258 \mathrm{bp}$; for APOC3, enzyme Sacl was used, the size of the digested fragments 292/209bp. The digested fragments of gene FABP2 and $A P O C 3$ were resolved using $4 \%$ agarose gel and then visualized on the Gel-Doc system.

\section{Statistical Analysis}

The biochemical parameters of the individuals in various groups were reported as means \pm standard deviation. To determine significant differences between the groups, analysis of variance test was used to compare biochemical parameters. Chi-squared test was applied to compare genotype distributions between the groups. For each genotype, Hardy-Weinberg equilibrium was tested with chi-squared goodness-of-fit test. All tests were performed using the SPSSPC software and Graph Pad.

\section{Results}

The comparison of age and biochemical parameters: age, fasting sugar levels, and lipid profile data of the DD group, NDD group, and $C$ group are given in - Table 1. There were statistically significant differences between the groups DD, NDD, and C group for FBS, CHOL, TG, LDL-C, and very-low density lipoprotein. No significant differences between groups were detected for age and HDL-C.

The distribution of genotypes and alleles of apo-C3 polymorphism between the DD, NDD, and C groups is reported in - Table 2. There was a significant difference in the geno- type distribution between the NDD and control groups (chisquare $=12$, d.f. $=2, p<0.001$ ). However, there was no significant difference in genotype distribution of DD and control groups (chi-square $=4.4$, d.f. $=2, p>0.001$ ). CC genotype was significantly more frequent in the control group compared with the DD and NDD group (70 vs. $46.46 \%)$. Conversely, GG genotype was more prevalent in the NDD (10\%) group compared with the DD (6.66\%), and no GG genotype was seen in C groups. However, CG genotype was more prevalent in the DD group (46.66\%), followed by the C group (30\%) and NDD group (20\%). There was a significant association of $A P O C 3 \mathrm{G}$ allele with hypertriglyceridemia $(p<0.05)$. Logistic regression analysis revealed APOC3 polymorphism to be significantly associated with dyslipidemia (odds ratio $[\mathrm{OR}]=2.6667,95 \%$ confidence interval $[\mathrm{CI}]=1.0510-6.7663, p=0.0341$; - Table 3).

The distribution of genotypes and alleles of FABP2 polymorphism between the DD, NDD, and C groups is reported in - Table 2. There was no significant difference in the genotype distribution between the DD, NDD, and $C$ groups (chi-square $=2.22$, d.f. $=2, p>0.001$ ). The distribution of AA, AG, and GG genotypes was found to be almost similar in all the three groups. No significant association of FABP2 A allele with hypertriglyceridemia was found $(p>0.05)$. Logistic regression analysis revealed no significant association of FABP2 polymorphism with dyslipidemia (OR $=0.4643,95 \%$ $\mathrm{CI}=0.1641-1.3136, p=0.1347 ;-$ Table 3 ).

\section{Discussion}

In the present pilot study, an unselected study population of 90 individuals consulting at AIIMS Bhopal Dyslipidemia Clinic (MP) was conducted, which is a cosmopolitan city with a diverse culture and different ethnic groups. Our aim was to identify genes associated with high serum levels of lipids in the NDD population by evaluating the correlation of polymorphism of each candidate gene to this condition. By the help of public databases namely PubMed (NCBI), we selected polymorphisms in two candidate genes-APOC3 (rs5128) and FABP2 (rs1799883)-since these polymorphisms were more prevalent in Indian population.

Table 1 Comparison of age and biochemical parameters between DD, NDD, and C patients

\begin{tabular}{|l|l|l|l|l|}
\hline Biochemical parameters & DD & NDD & C & $p$-Value \\
\hline Age & $52.27 \pm 11.00$ & $50.37 \pm 11.57$ & $49.73 \pm 11.27$ & 0.66 \\
\hline FBS & $180.36 \pm 68.56$ & $95.46 \pm 15.21$ & $95.76 \pm 12.68$ & $0.0001^{\mathrm{a}}$ \\
\hline TC & $225.53 \pm 92.82$ & $206 \pm 49.66$ & $158.8 \pm 38.2$ & $0.0004^{\mathrm{a}}$ \\
\hline TG & $222 \pm 97.03$ & $178.91 \pm 60.66$ & $100.03 \pm 25.8$ & $0.0001^{\mathrm{a}}$ \\
\hline LDL-C & $122.8 \pm 32.7$ & $125.46 \pm 43.7$ & $96.55 \pm 30.79$ & $0.0001^{\mathrm{a}}$ \\
\hline HDL-C & $45.25 \pm 26.65$ & $43.43 \pm 10.52$ & $42.46 \pm 15$ & 0.84 \\
\hline VLDL & $64.90 \pm 82.4$ & $48.53 \pm 54.44$ & $19.96 \pm 5.17$ & $0.0097^{\mathrm{a}}$ \\
\hline
\end{tabular}

Abbreviations: C, control; DD, diabetic dyslipidemics; FBS, fasting blood sugar; HDL-C, high-density lipoprotein cholesterol; LDL-C, low-density lipoprotein cholesterol; NDD, nondiabetic dyslipidemics; SD, standard deviation; TC, total cholesterol; TG, triglyceride; VLDL-C, very-low density lipoprotein cholesterol.

Data are reported as mean \pm SD.

${ }^{a} p<0.05=$ significant. 
Table 2 The distribution of genotypes and alleles of APOC3 and FABP2 polymorphism between the DD, NDD, and C groups

\begin{tabular}{|c|c|c|c|c|}
\hline \multicolumn{5}{|l|}{ APOC3 } \\
\hline Frequency & Genotype/Allele & $\begin{array}{l}\text { DD (Group 1) } \\
n=30\end{array}$ & $\begin{array}{l}\text { NDD (Group 2) } \\
n=30\end{array}$ & $\begin{array}{l}\text { C (Group 3) } \\
n=30\end{array}$ \\
\hline \multirow[t]{3}{*}{ Genotype } & GG & $2(6.66 \%)$ & $10(33.33 \%)$ & $0(0 \%)$ \\
\hline & CG & $14(46.66 \%)$ & $6(20 \%)$ & $9(30 \%)$ \\
\hline & CC & 14 (46.66\%) & 14 (46.66\%) & 21 (70\%) \\
\hline \multirow[t]{2}{*}{ Allele } & G & 0.3 & 0.43 & 0.15 \\
\hline & C & 0.7 & 0.57 & 0.85 \\
\hline \multicolumn{5}{|l|}{ FABP2 } \\
\hline Frequency & Genotype/Allele & $\begin{array}{l}\text { DD (Group 1) } \\
n=30\end{array}$ & $\begin{array}{l}\text { NDD (Group 2) } \\
n=30\end{array}$ & $\begin{array}{l}\text { C (Group 3) } \\
n=30\end{array}$ \\
\hline \multirow[t]{3}{*}{ Genotype } & $\mathrm{AA}$ & $10(33.33 \%)$ & $12(40 \%)$ & 11 (36.66\%) \\
\hline & AG & $9(30 \%)$ & $8(26.66 \%)$ & $13(43.33 \%)$ \\
\hline & GG & $11(36.66 \%)$ & $10(33.33 \%)$ & $6(20 \%)$ \\
\hline \multirow[t]{2}{*}{ Allele } & A & 0.48 & 0.53 & 0.58 \\
\hline & $\bar{G}$ & 0.52 & 0.47 & 0.42 \\
\hline
\end{tabular}

Abbreviations: APOC3; apolipoprotein C3; C, control; FABP2, fatty acid-binding proteins; DD, diabetic dyslipidemics; NDD, nondiabetic dyslipidemics. APOC3: $\mathrm{X}^{2}=12$, d.f. $=2,{ }^{*} p<0.05 ; \mathrm{FABP} 2: \mathrm{X}^{2}=2.22$, d.f. $=2, p>0.05$

Table 3 Logistic regression analysis of APOC3 (rs5128) and FABP2 (Ala54Thr) polymorphism and dyslipidemia

\begin{tabular}{|l|l|l|l|l|l|l|l|}
\hline \multirow{2}{*}{ Genes } & \multirow{2}{*}{ Genotype } & \multicolumn{2}{|l|}{ Group } & \multirow{2}{*}{ OR } & 95\% Cl & \multirow{2}{*}{-Value } \\
\cline { 3 - 5 } & & DD & NDD & C & & & \\
\hline \multirow{2}{*}{ APOC3 } & GG + CG & 16 & 16 & 9 & 2.6667 & $1.0510-6.7663$ & $0.0341^{*}$ \\
\cline { 2 - 5 } & CC & 14 & 14 & 21 & & & \\
\hline FABP2 & AA + AG & 19 & 20 & 24 & 0.4643 & $0.1641-1.3136$ & 0.1347 \\
\hline
\end{tabular}

Abbreviations: APOC3; apolipoprotein C3; C, control; Cl, confidence interval; FABP2, fatty acid-binding proteins; DD, diabetic dyslipidemics; NDD, nondiabetic dyslipidemics; OR, odds ratio.

* denotes that p-value is significant.

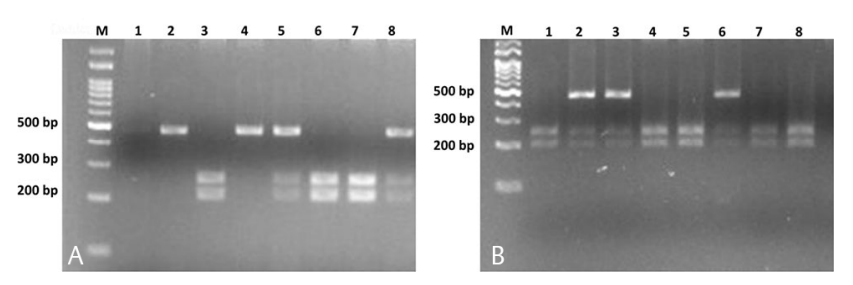

Fig. 1 Three types of banding pattern observed for apolipoprotein C3 $(A P O C 3)$ and fatty acid-binding proteins (FABP2) after restriction fragment length polymorphism (RFLP). $M=100$ bp ladder, 1-8 = digested polymerase chain reaction products. (A) Three types of banding pattern for gene FABP2 after RFLP-458 bp = wild type (GG genotype), 456/258/192 bp = heterozygous (GA genotype), 258/192 bp = homozygous (AA genotype). (B) Three types of banding pattern of gene $A P O C 3$ after RFLP-501 bp = wild type (CC genotype), $501 / 292 / 209$ bp $=$ heterozygous (GC genotype), 292/209 = homozygous (GG) genotype.

Polymorphisms in both genes have been associated with dyslipidemia and may pose a risk factor for CHD in future.

To our knowledge, this is the first study to test a significant association of APOC3 (rs5128) and FABP2 (rs1799883) polymorphism and abnormal serum lipid levels in NDD patients, but a comparable study was done to test the determinants of dyslipidemia in North Indians. ${ }^{9}$ The lipid profile values were highly variable among the population. Over $66 \%$ of the general adult population covered in this study have abnormalities in at least one of the lipid parameters. In the present study as expected, hypertriglyceridemia and hypercholesterolemia were most prevalent among DD group and NDD group and normal values were obtained for $C$ group. Similar observations in the age range of 35 to 70 years were also reported in one such study. ${ }^{10}$

For APOC3, we got three genotypes (-Table 2, - Fig. 1): CC (wild type), CG (heterozygous), and GG (homozygous). In DD group, $6.66 \%$ of the population was found to be homozygous, $46.66 \%$ of the population was found to be heterozygous, and $46.66 \%$ population had wild-type genotype. In NDD group, maximum polymorphism was found; $33.33 \%$ population was found to be homozygous, $20 \%$ of the population was found to be heterozygous, and $46.66 \%$ population had wild-type genotype. In $C$ group, the lowest polymorphism was found and no homozygotes were found; only $30 \%$ population was heterozygous and $70 \%$ population had wild-type genotype. In DD group and NDD group, it was found that homozygous 
individuals have significantly high TG and CHOL values (-Table 1), followed by heterozygotes; this indicates that genetic study is comparable to the biochemical findings, and there was a significant association of $A P O C 3 \mathrm{G}$ allele with hypertriglyceridemia $(p<0.05)$. Logistic regression analysis suggested that polymorphism in $A P O C 3$ gene is significantly associated with the occurrence of dyslipidemia $(\mathrm{OR}=2.6667$, 95\% CI = 1.0510-6.7663, $p=0.0341$; - Table 3).

Similar findings have been demonstrated that carriers of $G$ allele had higher levels of TG and CHOL in comparison to noncarriers. ${ }^{11}$ The overall frequency of occurring of GG genotype was found to be very low in the population followed by GC; similar observations were also suggested in the study. ${ }^{12}$ Thus, it can be hypothesized that polymorphism in APOC3 gene (GG genotype) is contributing to high TG and CHOL values in NDD group individuals in the study; similar results have been found in a multiethnic study conducted on US adults, which supports the fact that carriers of polymorphism have high TG and to CHOL levels. ${ }^{13}$

In the present study, investigations have clearly specified that mean value of TG in NDD group is $178 \pm 0.66 \mathrm{mg} / \mathrm{dL}$ as compared with the DD group with $222 \pm 97.03 \mathrm{mg} / \mathrm{dL}$ in clinically maintained cases with TG lowering medications.

Analysis of $A P O C 3$ gene can suggest here that DD group has high TG (mean value $222 \pm 97.03 \mathrm{mg} / \mathrm{dL}$ ) and $\mathrm{CHOL}$ $(225.53 \pm 92.82 \mathrm{mg} / \mathrm{dL})$, mainly due to diabetes mellitus, but they are also carriers of polymorphic allele (GC). In NDD group, high TG (178.9 $\pm 60.66 \mathrm{mg} / \mathrm{dL})$ and $\mathrm{CHOL}(206 \pm 49.66$ $\mathrm{mg} / \mathrm{dL}$ ) and maximum polymorphism indicates polymorphism in lipid metabolizing genes as one of the important factors. In C group, TG (100.03 $\pm 25.8 \mathrm{mg} / \mathrm{dL})$ and CHOL (158.8 $\pm 38.2 \mathrm{mg} / \mathrm{dL}$ ) were all in normal range. In $C$ group, the few polymorphisms are observed as carriers for the polymorphic allele (GC); they may also be at a risk of developing dyslipidemia in future.

For gene FABP2, we got three genotypes ( - Table 2, - Fig. 1): GG (wild-type), GA (heterozygous), and AA (homozygous). All three genotypes were found in all the three groups. All the groups contained an almost equal number of homozygotes, heterozygotes, and wild type genotype. In DD group, 33.33\% of the population was found to be homozygous, $30 \%$ of the population was found to be heterozygous, and $36.66 \%$ population had wild-type genotype. In NDD group, $40 \%$ population was found to be homozygous, $26.66 \%$ of the population was found to be heterozygous, and $33.33 \%$ population had wildtype genotype. In $C$ group for FABP2 gene, $36.66 \%$ of the population was found to be homozygous, $43.33 \%$ population was heterozygous, and $20 \%$ population had wild-type genotype in the present study. No significant association of FABP2 A allele with hypertriglyceridemia $(p>0.05)$ was found. Logistic regression analysis suggested no significant association of FABP2 polymorphism with dyslipidemia ( $\mathrm{OR}=0.4643,95 \%$ $\mathrm{CI}=0.1641-1.3136, p=0.1347$; - Table 3).

Previous study has demonstrated that genotypes AA in FABP2 have significantly high TG and CHOL levels followed by AG heterozygote that has been demonstrated in studies on elderly people of Croatian descent. ${ }^{14}$ Many studies have also suggested high prevalence of this polymorphism among all the other genes of lipid metabolism. ${ }^{15}$ Based on these observations, it may be possible to identify the risk groups in the studied population to predict the probability of developing vascular or cardiac disease based on studied gene. ${ }^{16}$

The study suggests that polymorphic alleles influence serum lipid levels and thus they may genetically predispose nondiabetic individuals to dyslipidemia. The results from our study agree with studies that correlate genetics, ethnicity, and environmental factors with dyslipidemia. ${ }^{17}$ Their results suggested that the APOC3 polymorphism was significantly associated with fasting plasma levels of $A P O C 3, \mathrm{TG}, \mathrm{TC}$, and LDL-C under the dominant model. The carriers of polymorphic allele had higher levels of TG, TC, and LDL-C than the noncarriers. A meta-analysis demonstrated that these polymorphisms are associated with CHD risk. ${ }^{18}$ The combination of these factors could explain the interindividual variations in lipid levels. Taken our results together, it may be possible that there is an association between the rs5128 and CHD. ${ }^{19}$ The clinical significance of possessing polymorphic allele has been demonstrated in some case-control studies that showed a two- to fivefold increase in frequency in patient groups with premature CHD and peripheral vascular disease compared with control groups. ${ }^{20,21}$ The knowledge of the molecular basis of dyslipidemia will allow not only their correct diagnosis but also advise them for routine check-ups and all the possible precautions to minimize the causes to develop CHD due to genetic dyslipidemia in nondiabetic individuals. Genetic diagnosis is helpful in the family investigation process, which allows early detection, therapeutic management, and subsequent reduction in dyslipidemia risk in such individuals as suggested by certain studies. ${ }^{22}$

\section{Conclusion}

In conclusion, the present study has strongly directed that there is difference in patterns of dyslipidemia in diabetic and nondiabetic population. The results mainly showed an association of $A P O C 3$ polymorphism with variation in serum lipid levels among the NDD group. The fact that even young adults are affected is distressing and thus screening from younger ages may help to promote lifestyle changes that can prevent or slow development of dyslipidemia for the population that comes under this group. Results of this study might facilitate in the understanding of the pathophysiology of dyslipidemia and multifactorial disorders like CHD in NDD patients. Since lipid levels may be genetically controlled, the identification of genetic variants linked with plasma lipid concentrations can provide beneficial information related to genotypephenotype relationships. These genetic variations may be useful in the development of diagnostic/prognostic markers for dyslipidemia in nondiabetic individuals. However, further studies with large sample size are required to validate the results from present study.

Authors' Contribution

Rashmi Chowdhary was involved in concepts, design, definition of intellectual content, literature search, clinical studies, experimental studies, data acquisition, data 
analysis, statistical analysis, manuscript preparation, manuscript editing, and manuscript review. Neha Masarkar was involved in design, definition of intellectual content, literature search, clinical studies, experimental studies, data acquisition, data analysis, statistical analysis, manuscript preparation, manuscript editing, and manuscript review. Sagar Khadanga was involved in concepts, design, clinical studies, data acquisition, and manuscript review. Rashmi Chowdhary has given guarantee for this manuscript.

\section{Statement of Approval}

This study has been approved by Institutional Human Ethics Committee.

\section{Funding}

This study is funded by Institutional Intramural Research Funding (LOP/2017/IM0147).

\section{Conflict of interest \\ Authors declare no conflicts of interest.}

\section{Acknowledgment}

Thanks to the Department of Biochemistry, Department of General Medicine, and the respected Director.

\section{References}

1 Stein R, Ferrari F, Scolari F. Genetics, dyslipidemia, and cardiovascular disease: new insights. Curr Cardiol Rep 2019;21(08):68

2 Ni WQ, Liu XL, Zhuo ZPet al.Serum lipids and associated factors of dyslipidemia in the adult population in Shenzhen. Lipids Health Dis 2015;14:71

3 Guptha S, Gupta R, Deedwania Pet al.Cholesterol lipoproteins and prevalence of dyslipidemias in urban Asian Indians: a cross sectional study. Indian Heart J 2014;66(03):280-288

4 Geldsetzer P, Manne-Goehler J, Theilmann Met al.Geographic and sociodemographic variation of cardiovascular disease risk in India: a cross-sectional study of 797,540 adults. PLoS Med 2018;15(06):e1002581

5 Burgess S, Harshfield E. Mendelian randomization to assess causal effects of blood lipids on coronary heart disease: lessons from the past and applications to the future. Curr Opin Endocrinol Diabetes Obes 2016;23(02):124-130

6 MihÄ ilÄ RG. Pragmatic analysis of dyslipidemia involvement in coronary artery disease: a narrative review. Curr Cardiol Rev 2019

7 Larifla L, Rambhojan C, Joannes MOet al.Gene polymorphisms of FABP2, ADIPOQ and ANP and risk of hypertriglyceridemia and metabolic syndrome in Afro-Caribbeans. PLoS One 2016;11(09): e0163421

8 Cui F, Li K, Li Y, Zhang X, An C. Apolipoprotein C3 genetic polymorphisms are associated with lipids and coronary artery disease in a Chinese population. Lipids Health Dis 2014;13:170

9 Chhabra S, Narang R, Krishnan LRet al.Apolipoprotein C3 SstI polymorphism and triglyceride levels in Asian Indians. BMC Genet 2002;3:9

10 Norata GD, Tsimikas S, Pirillo A, Catapano AL. Apolipoprotein C-III: from pathophysiology to pharmacology. Trends Pharmacol Sci 2015;36(10):675-687

11 Song Y, Zhu L, Richa M, Li P, Yang Y, Li S. Associations of the APOC3 rs5128 polymorphism with plasma APOC3 and lipid levels: a meta-analysis. Lipids Health Dis 2015;14:32

12 Smith CE, Tucker KL, Scott TMet al.Apolipoprotein C3 polymorphisms, cognitive function and diabetes in Caribbean origin Hispanics. PLoS One 2009;4(05):e5465

13 Ríos-González BE, Luévano-Ortega KE, Saldaña-Cruz AM, González-García JR, Magaña-Torres MT. Polymorphisms of seven genes involved in lipid metabolism in an unselected Mexican population. J Genet 2011;90(03):e114-e119

14 Auinger A, Helwig U, Rubin Det al.Human intestinal fatty acid binding protein 2 expression is associated with fat intake and polymorphisms. J Nutr 2010;140(08):1411-1417

15 Khattab SA, Abo-Elmatty DM, Ghattas MH, Mesbah NM, Mehanna ET. Intestinal fatty acid binding protein Ala54Thr polymorphism is associated with peripheral atherosclerosis combined with type 2 diabetes mellitus. J Diabetes 2017;9(09):821-826

16 Salto LM, Bu L, Beeson WL, Firek A, Cordero-Macintyre Z, De Leon M. The Ala54Thr polymorphism of the fatty acid binding protein 2 gene modulates HDL cholesterol in Mexican-Americans with type 2 diabetes. Int J Environ Res Public Health 2015;13(01): h13010052

17 Lakbakbi El Yaagoubi F, Charoute H, Morjane Iet al.Association analysis of genetic variants with metabolic syndrome components in the Moroccan population. Curr Res Transl Med 2017; 65(03):121-125

18 Li S, Guo YL, Zhao Xet al.Novel and traditional lipid-related biomarkers and their combinations in predicting coronary severity. Sci Rep 2017;7(01):360

19 Natarajan P, Kohli P, Baber Uet al.Association of APOC3 loss-offunction mutations with plasma lipids and subclinical atherosclerosis: the multi-ethnic Biolmage Study. J Am Coll Cardiol 2015;66(18):2053-2055

20 García-Giustiniani D, Stein R. Genetics of dyslipidemia. Arq Bras Cardiol 2016;106(05):434-438

21 Zhang H, Temel RE, Martel C. Cholesterol and lipoprotein metabolism: Early Career Committee contribution. Arterioscler Thromb Vasc Biol 2014;34(09):1791-1794

22 Chrispin J, Martin SS, Hasan RKet al.Landmark lipid-lowering trials in the primary prevention of cardiovascular disease. Clin Cardiol 2013;36(09):516-523 